\title{
Fetal Scalp Sampling
}

National Cancer Institute

\section{Source}

National Cancer Institute. Fetal Scalp Sampling. NCI Thesaurus. Code C114100.

A technique used to evaluate fetal acid-base status during labor by obtaining a capillary blood specimen from the fetus. 